\title{
L'engorgement des urgences menace la dignité des patients
}

\author{
Rick Mah, MD
}

English VERSION ON PAGE 365

$\mathrm{L}$ 'engorgement dans les urgences fait l'objet d'une étude approfondie pour évaluer ses causes et conséquences. Or, bien que sous-entendue, la menace à la dignité des patients posée par l'engorgement des urgences ne semble pas faire couler beaucoup d'encre. Le personnel soignant et les patients des services d'urgence perçoivent intuitivement cette menace, mais elle semble difficile à prouver, puisqu'il n'existe pas de définition universelle de la dignité ni de méthode pour la quantifier. Nous devons donc nous tourner vers d'autres secteurs, comme les soins palliatifs, les directives de pratique et les droits de la personne, pour tenter de définir et de mesurer l'impact de l'engorgement sur la dignité des patients. La dignité est au cœur de l'expérience humaine et, en tant que médecins, nous avons l'obligation morale, envers nos patients, nos collègues et nous-mêmes, de la préserver et de la promouvoir. La dignité des patients pourrait devenir un nouveau sujet de recherche dans la problématique de l'engorgement des urgences et une plus grande sensibilisation au problème contribuerait à améliorer les soins prodigués aux patients et leur satisfaction. Plus important encore, l'engorgement en tant que menace à la dignité des patients pourrait devenir un puissant incitatif pour mobiliser les gens, au-delà des services d'urgence à réagir face à cette crise.

\section{CONCEPTS DE BASE}

Le dictionnaire Oxford définit la dignité par « l'honneur et le respect que se mérite quelqu'un $\gg^{1}$ (adaptation libre). Dans le sens classique du terme, la dignité est attribuée à une personne qui se distingue ou qui excelle, tandis que selon la tradition judéo-chrétienne, chaque individu a une dignité intrinsèque puisqu'il a été créé « à l'image de Dieu ». Pour Kant, cette même valeur intrinsèque découle de notre autonomie et de notre rationnalité 2 . Après la Deuxième Guerre mondiale, la dignité a pris de l'importance, en devenant l'un des principes fondateurs de nombreuses constitutions nationales et déclarations internationales, dont la plus célèbre est probablement la Déclaration universelle des droits de l'homme ${ }^{3}$. Malgré le rôle central qu'elle occupe dans ces documents officiels et dans la société, la dignité se définit différemment selon les « cultures et les sous-cultures $\gg^{4}$. Le présent article abordera la dignité uniquement sous l'angle de la tradition occidentale.

\section{DIGNITÉ ET SOINS DE SANTÉ}

La dignité occupe une grande place dans de nombreux codes d'éthique régissant les professions et pourtant, on donne peu d'orientation concrète en la matière au professionnel ${ }^{5}$. Les analyses du concept laissent supposer que dans le contexte des soins de santé, la dignité fait appel aux valeurs « d'autonomie, de vérité, de justice et de respect des droits de la personne $\gg^{6}$ et se manifeste de diverses façons : « attention, conscience, respect des individus, dévouement, fraternité et défense active du patient $\gg^{7}$. Un article tiré de la littérature sur la médecine d'urgence définit comme suit les attributs de la dignité : respect, autonomie, habilitation et communication $^{8}$. Malheureusement, ces attributs souffrent de la même éphémérité que le terme qu'ils tentent de définir. Le but du présent article n'est pas de faire une dissertation abstraite, mais plutôt d'aborder la dignité dans ce qu'elle a de tangible et que l'on peut promouvoir, perdre, menacer ou enfreindre.

Service d'urgence, Hôpital Général de Montréal, Montréal (Québec)

Les opinions exprimées dans les éditoriaux sont celles des auteurs; elles ne reflètent pas nécessairement celles du JCMU ou de l'Association canadienne des médecins d'urgence. 


\section{MODÈLES FAVORISANT LA DIGNITÉ}

Les modèles de soins palliatifs et les directives de pratique qui préconisent la dignité tentent de combler le fossé entre les principes de protection de la dignité dans les soins et ce qui s'observe en réalité. Le modèle de protection de la dignité de Chochinov ${ }^{9}$ pour les soins palliatifs énumère les facteurs qui influent sur la dignité des patients et décrit une approche de préservation de la dignité dans le contexte clinique. Les secteurs où l'engorgement met en péril la dignité des patients en raison du manque d'espace ou de temps pour les soigner touchent la détresse occasionnée par des symptômes, notamment le piètre contrôle de la douleur ${ }^{10,11}$, l'atteinte à l'autonomie des patients, l'atteinte à leur intimitét ${ }^{12,13}$ et la teneur des soins ${ }^{14}$. Le National Health Service (NHS) du Royaume-Uni offre de son côté des directives de soins dans le respect de la dignité; on y énumère les facteurs et paramètres de l'intimité et de la dignité, de même que des indicateurs de promotion des pratiques optimales ${ }^{15}$. Et pourtant, l'expérience des unités de soins britanniques ${ }^{16}$ donne à penser que les recommandations visant la protection de l'intimité et de la dignité des patients peinent à s'appliquer dans un contexte pourtant plus propice que les urgences congestionnées.

\section{ATTEINTE À LA DIGNITÉ DANS LES URGENCES}

Selon Schachter, on reconnaît l'atteinte à la dignité humaine, même si ce terme abstrait est difficile à définir. « Je la reconnais quand je la vois, même si je ne peux vous la décrire ${ }^{17}$. » De son côté, Mann a proposé une taxonomie de quatre types d'atteintes à la dignité : 1) être ignoré ou trop peu reconnu; 2) être vu, mais uniquement comme un membre d'un groupe; 3) subir contre son gré l'envahissement de son espace personnel; et 4) subir l'humiliation ${ }^{18}$. Les études qualitatives qui ont porté sur l'expérience des patients dans les services d'urgence et leurs couloirs donnent à penser que l'engorgement menace la dignité parce qu'elle enfreint le principe d'intimité ${ }^{19}$ et que la teneur des soins est inappropriée $^{20,21}$. Or, le poids réel de ces études réside dans le récit des patients, puisque c'est à travers leurs propos que l'atteinte à leur dignité devient tangible. Dans le cas de la dignité, la généralisation des expériences humaines aux fins de l'établissement d'une preuve dilue la gravité de l'atteinte ressentie par l'individu, ce qui, en soi, selon la taxonomie de Mann, représente une atteinte à la dignité (c.-à-d., être vu, mais seulement comme membre d'un groupe). Ne reconnaissant pas l'atteinte à la dignité du patient en tant qu'individu, nous déshumanisons encore la crise dans nos urgences. Certains récits de patients peuvent pourtant nous éclairer :

« J'ai été hospitalisée pour des problèmes cardiaques, mais on m'a gardée sur une civière dans le couloir. Comme je ne pouvais pas me tenir debout, mon principal problème était d'utiliser la toilette. Ce n'est pas très agréable d'être assise sur la bassine dans un couloir ... J'étais là, à émettre de sons et des odeurs devant tout le monde ... c'était pénible et humiliant ${ }^{19}$. »

«Les premiers jours, j'avais l'impression de n'être personne, parce que je me trouvais dans le couloir. J'avais très mal et je ne me sentais pas bien du tout. Les gens et les infirmières passaient en coup de vent, sans me voir ... Nous étions ${ }^{11}$ dans ce couloir ${ }^{19}$. »

De son côté, une patiente âgée, incapable d'uriner dans la bassine à cause d'une position et d'un environnement peu propices, raconte : « [L'infirmière m'a dit] "Je n'ai pas le temps pour ça... je vous mets une couche ..." Cela m'a anéantie. La seule pensée d'avoir à mettre une couche et de me faire dire de "faire " [uriner] dans ma couche ... je veux dire, ils ne peuvent pas vous forcer. Ici, on vous force ... ${ }^{20}$.

\section{DIGNITÉ ET DROITS DE LA PERSONNE}

Un autre cadre dans lequel on peut étudier en quoi l'engorgement des urgences menace la dignité est celui des droits de la personne. La dignité étant l'un des concepts essentiels des droits de la personne, « ces droits peuvent être analysés et défendus comme nécessaires à la protection ou la promotion de la dignité humaine ${ }^{22} \gg$. Deux droits se trouvent compromis par l'engorgement des urgences; ce sont le droit à la santé et le droit de ne pas être soumis à la torture, ni à des peines ou traitements cruels, inhumains ou dégradants.

L'article 12 du Pacte international relatif aux droits économiques, sociaux et culturels reconnaît le droit qu'a toute personne de jouir du meilleur état de santé physique et mentale qu'elle soit capable d'atteindre ${ }^{23}$. Ce droit implique la protection contre « les atteintes à l'intégrité physique, une morbidité inutile et une mortalité qu'il serait possible de prévenir ${ }^{24} \gg$ et l'engorgement des urgences place probablement les patients à risque à l'égard de telles menaces. Il a été possible d'établir un lien entre l'engorgement et une réduction de la qualité des soins aux patients, par exemple une maîtrise sous-optimale de la douleur ${ }^{10,11}$ et des retards à prodiguer des traitements urgents (p. ex., thrombolyse pour un infarctus du myocarde $)^{25}$ ou à administrer des antibiotiques pour la pneumonie ${ }^{26,27}$. On a également établi un lien entre l'engorgement et un pronostic plus sombre, par exemple une augmentation des complications cardiovasculaires ${ }^{28,29}$, un risque élevé de pneumonie ${ }^{30}$ 
et un séjour prolongé $e^{31,32}$. D'autres études ont donné à penser que l'engorgement des services d'urgence peut accroître le risque de mortalitééc-35. Le droit à la santé inclut en outre le droit à la prophylaxie et au traitement des maladies, ainsi qu'à la lutte contre celles-ci ${ }^{23}$ et l'engorgement accroît le risque de transmission des maladies contagieuses en raison du manque de personnel ${ }^{36}$ et de la promiscuité entre les patients ${ }^{37}$. L'un des exemples les plus graves de ce risque est l'épidémie du syndrome respiratoire aigu sévère (SRAS) où « la présence d'un seul patient dont le SRAS n'était pas reconnu dans un service d'urgence congestionné a constitué l'épicentre d'une éclosion ${ }^{38} \gg$.

L'article $7 \mathrm{du}$ Pacte international relatif aux droits économiques, sociaux et culturels interdit la torture et les traitements ou punitions cruels, inhumains ou dégradants $^{39}$. Parmi les méthodes de torture reconnues, on retrouve les températures extrêmes, la privation de sommeil, le bombardement sensoriel de bruit ou de lumière, l'absence d'intimité et les gestes d'humiliation ${ }^{40,41}$. Bien que bon nombre, sinon la totalité, de ces conditions existent jusqu'à un certain point dans les services d'urgence, l'engorgement en soi n'est pas une torture, puisque la définition d'une torture requiert que le traitement soit intentionnel et qu'il cause une souffrance importante ${ }^{42}$. L'engorgement pourrait toutefois être considéré comme étant cruel, inhumain ou dégradant et contrevenir ainsi aux normes visant à protéger les populations vulnérables. Le projet Sphere ${ }^{43}$ recommande d'allouer à chaque personne au moins $3,5 \mathrm{~m}^{2}$ de surface pour protéger sa dignité dans les camps de réfugiés. Dans les urgences engorgées, la surface par patient avoisine les $2 \mathrm{~m}^{2}$ lorsque les civières sont placées côte à côte. Les Conventions de Genève, qui visent à protéger les prisonniers de guerre et les civils en temps de guerre, précisent que les pièces de vie doivent être adéquatement éclairées, chauffées et spacieuses et que la nuit, les gens « bénéficieront de l'obscurité propre à assurer leur

\begin{tabular}{|c|c|c|}
\hline Menace & Facteurs & Exemples \\
\hline \multirow{10}{*}{$\begin{array}{l}\text { Traitement cruel, inhumain et } \\
\text { dégradant : ne répond pas aux normes } \\
\text { minimales }\end{array}$} & \multirow[t]{5}{*}{ Abri } & Espace personnel insuffisant \\
\hline & & Température ambiante inadéquate \\
\hline & & Excès de bruit et de lumière \\
\hline & & Privation de sommeil \\
\hline & & Actes d'humiliation \\
\hline & Nourriture et eau & Accès inadéquat \\
\hline & \multirow[t]{2}{*}{ Hygiène personnelle } & Accès inadéquat \\
\hline & & Actes d'humiliation \\
\hline & \multirow[t]{2}{*}{ Securité } & Risque de préjudice \\
\hline & & Détresse phsychologique \\
\hline \multirow[t]{8}{*}{ Menace au droit à la santé } & \multirow[t]{2}{*}{ Souffrance non justifiée } & $\begin{array}{l}\text { Détresse liée à des symptômes (p. ex., gestion } \\
\text { inadéquate de la douleur) }\end{array}$ \\
\hline & & Détresse psychologique \\
\hline & \multirow[t]{3}{*}{ Morbidité évitable } & $\begin{array}{l}\text { Retard de traitements urgents (p. ex., thrombolyse, } \\
\text { antibiotiques) }\end{array}$ \\
\hline & & $\begin{array}{l}\text { Pronostics sombres (p. ex., complications } \\
\text { cardiovasculaires, risque accru de pneumonie) }\end{array}$ \\
\hline & & Hospitalisation prolongée \\
\hline & Mortalité évitable & Risque accru de mort \\
\hline & \multirow{2}{*}{$\begin{array}{l}\text { Transmission de maladies } \\
\text { infectieuses }\end{array}$} & SARM \\
\hline & & SRAS \\
\hline \multirow{6}{*}{$\begin{array}{l}\text { Manquement à la protection et à la } \\
\text { promotion de la dignité }\end{array}$} & \multirow[t]{4}{*}{ Teneur des soins inappropriée } & Patient ignoré ou trop peu reconnu \\
\hline & & $\begin{array}{l}\text { Manque de respect ou de compréhension à l'égard du } \\
\text { patient }\end{array}$ \\
\hline & & Actes d'humiliation \\
\hline & & $\begin{array}{l}\text { Manquement à la promotion de l'autonomie du patient } \\
\text { (paternalisme, absence de consentement éclairé) }\end{array}$ \\
\hline & \multirow[t]{2}{*}{ Violations de l'intimité } & Violations de la confidentialité \\
\hline & & Modestie du patient non respectée \\
\hline
\end{tabular}


repos dans les meilleures conditions $\gg^{44,45}$. Des normes similaires sont en vigueur pour protéger les prisonniers civils $^{46}$. Si l'on applique ces normes minimums comme point de référence, l'engorgement des services d'urgence place les patients à risque à l'égard de traitements inhumains et dégradants et, par le fait même, menace leur dignité (tableau 1).

\section{DIRECTIONS FUTURES}

La médecine d'urgence pourrait tirer d'importantes leçons de l'abondance des écrits sur la santé et les droits de la personne et sur la médecine humanitaire. Une plus grande sensibilisation aux facteurs qui menacent la dignité des patients permettrait d'améliorer la communication, de promouvoir l'autonomie et de corriger les problèmes d'intimité, en partie par le réaménagement et la réorganisation des services d'urgence. L'impact de l'engorgement sur la dignité des patients doit faire l'objet d'études directes plutôt que d'extrapolations à partir de sources ne concernant pas directement les salles d'urgence. Il faut arriver à mesurer le paramètre « dignité » aux services d'urgence à partir des modèles établis par Chochinov et le British National Health Service, mais également, sur une base plus quantifiable, au moyen de paramètres liés à la santé (p. ex., le droit à la santé) et à des normes minimales. En s'attaquant au problème de la protection de la dignité dans le contexte des services d'urgence au sens large, comme le décrit le présent article, non seulement la santé des patients s'améliorera-t-elle probablement, mais aussi leur satisfaction sera plus grande.

\section{CONCLUSION}

En dépit du fait qu'il s'agit d'un concept abstrait, la dignité peut devenir un puissant moteur de changement en raison de son universalité et de son pouvoir unificateur, mobilisateur et personnalisateur. Les professionnels de la santé et les administrateurs verront la nécessité d'agir en prenant conscience que l'engorgement est perçu comme une crise humanitaire ou un problème grave de santé publique plutôt que comme un simple problème d'urgence. La dimension des droits de la personne est plus accessible au public et contribuera à consolider son appui envers des changements de politiques publiques visant à combattre l'engorgement. À titre de fournisseurs de soins de santé aux services d'urgence, nous ne pouvons nous permettre de négliger le caractère humain de nos interventions et nous ne pouvons pas non plus laisser les établissements ou la société oublier cette dimension.

Remerciements : L'auteur tient à remercier les $\mathrm{D}^{\text {rs }} \mathrm{Scott}$ Delaney, Alison Doucet, Kenneth Doyle et Kathleen Glass pour leurs commentaires, leur soutien et leur encouragement inestimables.

\section{Conflits d'intérêts : aucun déclaré.}

Mots clés : service d'urgence, urgences, engorgement, dignité, droits de la personne

\section{RÉFÉRENCES}

1. Soanes C, Stevenson A, Eds. The Oxford Dictionary of English. Oxford (UK): Oxford University Press; 2005.

2. Schulman A. Bioethics and buman dignity. President's Council on Bioethics: Staff Working Paper. 2005. Disponible à : www.bioethics.gov/background/human_dignity.html (consulté le 20 mai 2009).

3. Déclaration universelle des droits de l'homme, AGNU, résolution 217A, 3e session, 1948.

4. Shultziner D. Human dignity - functions and meanings. Global Jurist Topics 2003;3:3. Disponible à : www.bepress.com /gj/topics/vol3/iss3/art3 (consulté le 20 mai 2009).

5. Association médicale canadienne. Code de déontologie de l'AMC [politique. Ottawa (Ont.) : L'Association; 2004. Disponible à : http://policybase.cma.ca/dbtw-wpd/PolicyPDF/PD04-06F.pdf (consulté le 20 mai 2009).

6. Coventry ML. Care with dignity: a concept analysis. 7 Gerontol Nurs 2006;32:42-8.

7. Soderberg A, Gilje F, Norberg A. Dignity in situations of ethical difficulty in intensive care. Intensive Crit Care Nurs 1997;13:135-44.

8. Griffin-Heslin VL. An analysis of the concept of dignity. Accad Emerg Nurs 2005;13:251-7.

9. Chochinov HM. Dignity-conserving care - a new model for palliative care. JAMA 2002;287:2253-60.

10. Hwang U, Richardson L, Sonuyi T, et al. The effect of emergency department crowding on the management of pain in older adults with hip fracture. 7 Am Geriatr Soc 2006;54: 270-5.

11. Pines JM, Hollander J. Emergency department crowding is associated with poor care for patients with severe pain. Ann Emerg Med 2008;51:1-5.

12. Karro J, Dent AW, Farish S. Patient perceptions of privacy infringements in an emergency department. Emerg Med Australas 2005;17:117-23.

13. Moskop JC, Marco CA, Larkin GL, et al. From Hippocrates to HIPAA: privacy and confidentiality in emergency medicine - part II: challenges in the emergency department. Ann Emerg Med 2005;45:60-7.

14. Larsen B, Skretting P, Farstad T. Patients in corridors consequences for quality of care and patient integrity [article in Norwegian]. Tidsskr Nor Laegforen. 2000;120:2636-8.

15. Essence of care. Patient-focused benchmarks for clinical governance. London (UK): Department of Health; 2003. p. 119-128. Disponible à : www.dh.gov.uk/en/Publicationsandstatistics /Publications/PublicationsPolicyAndGuidance/DH_4005475 ?IdcService=GET_FILE\&dID $=29341 \&$ Rendition $=$ Web (consulté le 20 mai 2009).

16. Woogara J. Patients' rights to privacy and dignity in the 
NHS. Nurs Stand 2005;19:33-7.

17. Schachter O. Human dignity as a normative concept. Am J Int Law 1983;77:848-54.

18. Mann J. Dignity and health: The UDHR's revolutionary first article. Health Hum Rights 1998;3:30-8.

19. Foss C, Krogstad U. Invisible patients - experiences of lying in a corridor [article in Norwegian]. Tidsskr Nor Laegeforen 2000;120:2639-41.

20. Coughlan M, Corry M. The experiences of patients and relatives/significant others of overcrowding in accident and emergency in Ireland: a qualitative descriptive study. Accad Emerg Nurs 2007;15:201-9.

21. Wellstood K, Wilson K, Eyles J. "Unless you went in with your head under your arm": patient perceptions of emergency room visits. Soc Sci Med 2005;61:2363-73.

22. Chaskalson A. Human dignity as a constitutional value. In: Kretzmer D, Klein E, Eds. The concept of buman dignity in buman rights discourse. Netherlands: Kluwer Law International; 2002. p. 133-44.

23. Pacte international relatif aux droits civils et politiques, résolution 2200A, AGNU, 21e session, 1966. Disponible à : www .unhchr.ch/french/html/menu3/b/a_cescr_fr.htm. (consulté le 20 mai 2009).

24. Le droit au meilleur état de santé susceptible d'être atteint. Questions de fond concernant la mise en œuvre du pacte international relatif aux droits économiques, sociaux et culturels, OG 14, Conseil économique et social. Comité des droits économiques, sociaux et culturels, 22e session, 2000, E/C.12/2000/4.

25. Schull MJ, Vermuelen M, Slaughter G, et al. Emergency department crowding and thrombolysis delays in acute myocardial infarction. Ann Emerg Med 2004;44:577-85.

26. Fee C, Weber E, Maak C, et al. Effect of emergency department crowding on time to antibiotics in patients admitted with community-acquired pneumonia. Ann Emerg Med 2007; 50:501-9.

27. Pines JM, Locallo AR, Hollander J, et al. The impact of emergency department crowding measures on time to antibiotics for patients with community-acquired pneumonia. Ann Emerg Med 2007;50:510-6.

28. Pines JM, Hollander J. The impact of emergency department crowding on cardiac outcomes in ED Patients with potential acute coronary syndromes [résumé]. Ann Emerg Med 2007;50:S3.

29. Dierks DB, Roe M, Chen A. Prolonged emergency department stays of non-ST-segment elevation myocardial infarction patients are associated with worse adherence to the American College of Cardiology/American Heart Association Guidelines for Management and Increased Adverse Events. Ann Emerg Med 2007;50:489-96.

30. Carr BG, Kaye A, Wiebe D, et al. Emergency department length of stay: a major risk factor for pneumonia in intubated blunt trauma patients. 7 Trauma 2007;63:9-12.

31. Liew D, Liew D, Kennedy MP. Emergency department length of stay independently predicts excess inpatient length of stay. Med J Aust 2003;179:524-6.

32. Richardson DB. The access-block effect: relationship between delay to reaching an inpatient bed and inpatient length of stay. Med J Aust 2002;177:492-5.
33. Sprivulis PC, Da Silva JA, Jacobs IG, et al. The association between hospital overcrowding and mortality among patients admitted via Western Australian emergency department. Med J Aust 2006;184:208-12.

34. Richardson DB. Increase in patient mortality at 10 days associated with emergency department overcrowding. Med 7 Aust 2006;184:213-6.

35. Chalfin DB, Trzeciak S, Likourezos A, et al. Impact of delayed transfer of critically ill patients from the emergency department to the intensive care unit. Crit Care Med 2007; 35:1477-83.

36. Henderson DK. Managing methicillin-resistant staphylococci: a paradigm for preventing nosocomial transmission of resistand organisms. Am 7 Med 2006;119(6A):S45-52.

37. Kibbler CC, Quick A, O'Neill AM. The effect of increased bed numbers on MRSA transmission in acute medical wards. 7 Hosp Infect 1998;39:213-9.

38. Schull MJ, Redelmeier D. Infection control for the disinterested. CMAJ 2003;169:122-3.

39. International Covenant on Civil and Political Rights, Res. 2200A, UNGA, 21st Sess., 1966. Available: www.unhchr.ch/html /menu3/b/a_ccpr.htm. (accessed 2009 May 20).

40. Leave no marks: enhanced interrogation techniques and the risk of criminality. Cambridge (MA): Physicans for Human Rights; 2007. Available: http://physiciansforhumanrights.org/library /report-2007-08-02.html (accessed 2009 May 20).

41. Interrogations, torture and ill treatment: legal requirements and bealth consequences. Cambridge (MA): Physicians for Human Rights; 2004. Disponible à : http://physiciansforhumanrights .org/library/documents/reports/iraq-medical-consequences-of .pdf (consulté le 20 mai 2009).

42. Convention contre la torture et autres peines ou traitements cruels, inbumains ou dégradants, résolution 39, AGNU, 46e session, 1984. Disponible à : www.unhchr.ch/french/html/menu3/b /h_cat39_fr.htm (consulté le 20 mai 2009).

43. Sphere bandbook humanitarian charter and minimum standards in disaster response. The Sphere Project; 2004. Disponible à : www.sphereproject.org/handbook/pages/navbook.htm?para $\mathrm{m} 1=0$ (consulté le 20 mai 2009).

44. Comité international de la Croix-Rouge. Convention de Genève relative à la protection des personnes civiles en temps de guerre. Genève (Suisse) : le Comité; 1949, article 85. Disponible à : www.icrc.org/dih.nsf/FULL/380?OpenDocu ment (consulté le 20 mai 2009).

45. Comité international de la Croix-Rouge. Convention de Genève relative au traitement des prisonniers de guerre. Genève (Suisse) : le Comité; 1949, article 25 P3. Disponible à : http:// www.icrc.org/dih.nsf/FULL/375? OpenDocument (consulté le 20 mai 2009).

46. Premier Congrès des Nations Unies pour la prévention du crime et le traitement des délinquants. Ensemble de règles minima pour le traitement des détenus, résolution $663 \mathrm{C}, \mathrm{NU}, 24 \mathrm{e}$ session, 1955. Disponible à : www.unhchr.ch/french/html/menu3/ b/h_comp34_fr.htm (consulté le 20 mai 2009).

Correspondance : $\mathrm{D}^{\mathrm{r}}$ Rick Mah, Hôpital Général de Montréal, 1650 avenue Cedar, Montréal QC H3G 1A4; rick.mah@mcgill.ca 